mentales adultas y la asunción de roles adultos, lo que puedc suponer instalarse en la transitoriedad o en una posición ansiogena o de frustración, pero no un cambio radical.

El octavo capítulo aborda la condición docente. Aunque existe una gran diferencia entre un catedrático de universidad y un profesor de $\mathrm{EGB}$, hay una tendencia --siendo el sector más castigado el de enseñanzas meóias- hacia la desprofesionalización del enseñante (a la pérơida de competencia, independencia y autorregulación), es (ecir, hacia su proletarización. Ha perdido el control sobre los medios, el objetivo y el proceso de su trabajo. Así, las regulaciones determinan qué enseñar y limitan con ello la gama de métodos posibles, hay funciones que se atribuyen a trabajadores cspecíficos, a los editores, etc. Concluye señalando que el colectivo de enseñantes se encuer.tra entre los profesionales y los trabajadores proletarizados, es decir, en el lugar de las semiprofesiones. Fnresumen, autonomía a cambio de bajos salarios.

Por último, en el noveno capítulo, aborda la reforma de la enseñanza y señala que se hia convertido en un sindrome permanente de nuestra sociedad porque reformar la educación es la mancra de no reformar nada que forme parte esencial del orden establectdo (salario, relaciones de propiedad o distribución de ésta, etc.) y porque hay un desajuste entre igualdad de oportunidades y reforma educativa. Señala las dificultades a que se enfrenta toda innovación o reforma: los métodos y pautas organizativas existentes, las tradiciones heredadas y las plantillas de enseñantes (resulta muy difícil introducir contenidos nuevos porque es muy difícil librarse o reducir el espacio de los viejos). Finaliza señalando que, dado el espacio de autonomía del profesor dentro del aula, las innovaciones sólo pueden tener éxito si surgen de los mismos profesores, son asumidas por éstos o son estimuladas por la administración. De lo contrario, significan un salto en el vacío que pone en cuestión la autoridad y profesionalidad del profesor.

Jordi Solbes Matarredona

\title{
PUBLICACIONES RECIBIDAS
}

\section{MANUAL BIBLIOGRAFIC PER A L'ENSENY AMENT DE LA FILOSOFIA}

Garcia Raffi, X. i Hernández, F.J. Servei de Formacio Permanent de la Universitat de València.

Illustre Collegi de Doctors i Llicen-

Dentro de su labor de apoyo a la formacion del profesorado, la colaboración de ambas instituciones -el Servei de Formacio Permanent de la Universitat de València y el Il.lustre Col.legi de Doctors i Llicenciats de València- han editado esta amplia compilación de libros de texto, Kibros y artículos acerca de la Didáctica de la Filosofía, revistas filosóficas y pedagógicas relacionadas con dicha materia y un extenso repertorio de direcciones de interés para la enseñanza de la Filosofía.

La información que recogen las $\$ 5$ páginas de la obra ha de resuitar un recurso imprescindible para el profesorado de Filosofía y una importante fuente de consulta para el profesorado de Ciencias por la abundancia de información que contiene. ciats de Valencia.Valencia. 1990.

La obra es casi exhaustiva, aunque no figuran los trabajos del Seminari Permanent de Fìlosofia deI ICE de la UAB, ni los del Grup de Filosofia del Casal del Mestre de Santa Coloma, que a buen seguro figlırarán en futuras ediciones. Esto no debe desmerecer el considerable esfuerzo lizvado a cabo y que viene a cubrir partedel cnome déficit en repertorios bibliográficos tenáticos que aqueja at mercado español.

Para cualquier información dirigirse a: Francesc Jesús Hernández i Dobon c/Litògraf l'asqual i Abat, 27-13 46017. Valincia. Tel. 963571906 .

\section{EXPERIENCIAS DE CIENCIAS}

Sam Ed Brown. Narcea. 1991.

\section{LA PERCEPCION DEL OLOR}

Duchesne, J. y Jaubert, J.N. Narcea. 1990.

No es habitual prestar excesiva atención, en las colecciones destinadas al profesorado de educación infantil, a los temas relacionados con la enseñanza de las ciencias y mucho menos, caso de hacerlo, superar la simple recopilación de experiencias.

Es por eso que debemos celebrar la aparición de estos dos volúmencs, en la colección «Primeros Años», que unen la revisión de conceptos teóricos con la reflexión didáctica de las diversas experiencias propuestas.

La primera de las obras consta de una interesante primera parte dedicada a analizar las relaciones de los niños y niñas con las ciencias, ofreciendo al profesorado un marco teórico general de actuación que se aplica más adelante en las sesenta y dos experiencias de que consta la obra. 
Cada una de estas experiencias forma parte de uno de los nueve bloques en que pueden agruparse (aire, animales, medio ambiente,plantas, sentidos, agua y experiencias variadas). En cada caso se especifica qué aprende el alumno, el material necesario, qué debe hacer el profesor/ay de qué debe hablar en clase.

La percepción del olor trata de forma monográfica todos los aspectos relacionados con el mundo de los olores y el sentido del olfato.

Fn la primera parte, teórica, se hace una descripción del sistema olfativo y una breve recopilación de vocabulario del tema. Más adelante, se da información sobre sustancias olorosas y algunos criterios para clasificarlas.

I.a segunda parte aborda una serie de cuestiones relacionadas con las actividades de enseñanza-aprendizaje que pueden plantearse en el tema (diversidad, vocabulario...) y se analiza el papel del olfato en la vida cotidiana. La breve descripción de las relaciones de algunas profesiones con el mundo de los olores supone una incursión en el complejo tema de la orientación vocacional y profesional, lo cual supone una novedad en las obras dedicadas a este nivel educativo.

En la parte final del libro, se proponen actividades prácticas que giran alrededor de nueve temas principales. Para cada una de las actividades se sigue el mismo esquema que en la obra antes citada.

Por la claridad de la exposición y por la riqueza de las orientaciones facilitadas, creemos que se trata de dos obras altamente recomendables.

Jordi Roig

\section{APRENDIENDO EN EL LABORA- TORIO}

Posada, J.M. Departamento de Física y Química. Colegio Miguel de Cervantes. Sao Pauld.

"Aprendiendo en el laboratorio" es un cuaderno que recoge catorce actividades experimentales de química. El interés de Ia obra radica en la decidida incorporación de la V de Gowin a cada actividad, con la finalidad de llevar a cabo una síntesis entre los conocimientos téricos y el trabajo experimental realizado. Para conseguir tan loable intención se ha incorporado, al inicio de la obra, una clara explicación acerca de la elaboración de diagramas en V. Una colección de cuestiones cualitativas pretende facilitar la aplicación del conocimiento adquirido, al final de cada experiencia, lo que posibilita, además, su evaluación. La obra finaliza con una ampliación de la información, dirigida al profesorado, acerca del aprendizaje significativo y de la realización de diagramas en $\mathrm{V}$.

R.V.

\section{MUDANCA COCEPTUAL NA SALA DE AULA}

Vaz Moniz dos Santos, M.E. Biblioteca do Educador. Livros Horizonte. 1991

Brasil, como antos otros países, se encuentra en pleno proceso de reforma educativa. Esta simultaneidad con nuestra situacion hace doblemente oportuno un libro como éste, en que se pretende ofrecer una sóljda fundamentación epistemológica a tina propuesta de pedagogía del cambio conceptual.

A partir de la roción de representación, objeto de importantes trabajos en diferentes disciplinas, se consideran las características de una enseñanza que quiera hacer conscientes a los alumnos de sus concepciones alternativas y pretenda implicarlos activamente en el proceso de cambio conceptual.

Una profundización en el debate abierto por la epistemología racionatista contemporánea, una sistematización y una reflexión acerca de las tendencias y resultados de la investigación sobre las concepciones al"ernativas de los alumnos, son las bases de las ideas de fuerza que articulan el discurso de la autora.

El texto puede servir de marco a una discusión profundia y bien fundamentada sobre ta problem.ítica educativa.

J.G.

\section{TREBALLS DEL MUSEL DE GEOLOGIA}

\section{Ajuntament de Barcelona.}

Se trata del primer volumen de una nue. va colección dedicada a la divulgación de trabajos directa o indirectamente relacionados con las actividades del museo.

La revista, de hecho un libro por su volumen y formato, está profusamente ilustrada y editada con sumo cuidado. Recoge once artículos de gran calidad y erudición, fruto del trabajo del personal del propio museo y de colaboraciones provinentes de diversas universidades españolas (Barcelona, UAM, Sevilla) y extranjeras (Berna, Toulouse y Córdoba [Argentina]).

Aparte del artículo inicial en que pasa revista a la primera fase de la historia del museo (1878-1905), los trabajos pueden agruparse en tres bloques diferenciados, que podríamos llamar palcontológico, geológico y museográfico, atendiendo a la temática que desarroltan.

Entre los artículos del primer bloque figuran las descripciones extensas y muy documentadas de algunos fósiles de cetáceos e insectos relacionados con los fondos del museo. Un análisis de los bivalvos del Bajo Guadalquivir, la síntesis de estudios sobre depósitos sedimentarios argentinos, y una aproximación a la historia de los cocodrilos enanos son otros temas abordados en este blogue.

Dos artículos constituyen el segundo bloque. El primero de ellos analiza las relaciones entre la sierra del Cadí y la zona del Pedraforca, aportando datos en favor de una nueva hipótesis. In el segundo, se recogen estudios de los yacimientos de Almadén y Entredicho.

La informatización de los datos de colecciones y la gestión de una cartoreca son objeto de reflexión de los últimos artículos de este volumen.

I,a revista tiene periodicidad anual. I,a Biblioteca del Museu de Geologia, sita en el Parc de la Ciutadella, s/n. 08003 Barcelona, facilita todo tipo de información acerca de esta publicación, a la que damos la bienvenida desde estas páginas.

Jordi Roig 


\section{CIENCIA Y RELIGIÓN}

López, S. i de la Fuente, P. Casal del Mestre. Sunta Coloma de Gramenet.1991.

Son pocas las ocasiones en las que el profesorado de ciencias propone actividades al alumnado destinadas a la formación de actitudes, y más limitadas aún și el tema se sitúa en los límites de lo que la propia ciencia estudia. En esas contadas ocasiones, la programación se hace difícil por la escasez de obras que faciliten recursos en este sentido.
El Grup de Filosofia del Casal del Mestre de Santa Coloma ha animado la confección de diversas recopilaciones, pensadas cesde un punto de vista didáctico, que pueden situarse en la frontera entre la Ciencia y la Filosofía con la intención de cubrir parte de este déficit.

La obra que nos ocupa es, fundamentalmente, una antología de dicz textos breves de Albert Einstein, acompañados por una serie de cuestiones referidas a cada texto y una colección de cuestiones de reflexión, cuyo tema central es la relación de la ciencia con la religión. La multitud de cuestiones y la riqueza de matices que pueden surgir al tratarlas en clase permiten considerar este breve texto, -76 páginas-como un recurso de gran utiłidad.

Para cualquier información dirigirse a: Grup de Filosofia.

Casal del Mestre

c/ Sants, 1 baixos

08924. Santa Coloma de Gramenet.

R.V.

\section{TESIS DIDÁCTICAS}

DISEÑO, APLICACIÓN Y EVAIUACION DE MODULOS DE APRENDIZAJE PARA LA FORMACIÓN INICIAL DEL PROFESORA. DO DE EGB DE CIENCIAS

\section{Tesis doctoral}

Autora: Emigdia Repetto Jiménez

Director: Dr. Antonio de Pro Bueno

Lugar: Departamento de Didácticas Es-

peciales. Universidad de La Laguna

Esta tesis se enmarca en la problemática de la formación inicial del profesorado de ECiB de Ciencias.

A partir de algunos interrogantes y conclusiones obtenidos en experiencias anteriores se consider 6 la necesidad de un cambio en el proceso de formación de estos profesores y se plantearon una serie de cuestiones que demandaban la incorporación de determinadas estrategias de aprendizaje de la Didáctica de la Física y de la Química. La muestra estuvo constituida por los alumnos de esta asignatura en la Escuela Universitaria de Profesorado de EGB de Las Palmas.

Tres eran los problemas principales: a) ¿Cuál es la situación inicial de los futuros profesores de EGB de Ciencias cn cuanto a su formación científica y su formación profesional? b) ¿Cuál es la incidencia de las metodologías aplicadas en el Módulo I (Estudio del sonido); Módulo II (Estudio de la Electricioad), y Módulo III (Estudio de las Reacciones Químicas) en la formación inicial de los futuros profesores de EGB de Ciencias? c) ¿Qué incidencia tiene la metodología desarrollada en la investigación sobre la práctica docente de estos alumnos?

Sc hizo, lógicamente, una revisión de los trabajos, estudios e investigaciones recogidos en la literatura científica y que configuran el marco teórico al que referir las hipotesis experimentales.

La Hipótesis Principal Uno, estudia las condiciones iniciales de los alumnos. Se diseñaron unas pruebas y encuestas que sirvieron para determinar las características de los alumnos en función de sus conocimientos científicos y profesionales. Para la Hipótesis Principal Dos, se diseñaron tres móduIos de aprendizaje, escalonándose los contenidos didácticos a lo largo de los mismos. En la Hipótesis Principal Tres se proyectaron experiencias sobre el tópico de las Reacciones Químicas en EGB.

Por otro Iado, se idearon una serje de pruebas e instrumentos que nos facilitaran el análisis cualitativo y cuantitativo de las variables: prueba inicial y final, encuestas, cuestionarios, protocolos de observación sistemática... Se aplicaron técnicas estadísticas en el tratamiento de los datos (Programa BMD P4M. Vari$\max$.

Globalmente se ha creado una cstructura de investigación que pretende integrur diferentes paradigmas y tópicos educativos que tienen especial relevancia en la formación inicial de los profesores de EGB de Ciencias.

LOS TRABAJOS PRÁCTICOS EN LA ENSENANZA DE LA KISICA Y OLIMICA: UN ANÁLISIS CRÍTICO Y UNA PROPUESTA FUNDAMENTADA

\section{Tesis doctoral}

Autor: José Payá Peris

Directores: Drs. D. Daniel Gil Pérez y D. Jaime Carrascosa Alís

I ugar: Departament de Diddctica de les Ciències Experimentals. Universitat de Valencia.

Fecha: Junio de 1991.

Este trabajo se enmarca dentro de una línea de investigación orientada hacia la 